\title{
A SEPARABLE SOMEWHAT REFLEXIVE BANACH SPACE WITH NONSEPARABLE DUAL
}

\author{
ROBERT C. JAMES ${ }^{1}$
}

Communicated by Jacob Feldman, November 18, 1973

\begin{abstract}
An example is given of a separable Banach space $X$ whose dual is not separable, but each infinite-dimensional subspace of $X$ contains an infinite-dimensional subspace isomorphic to Hilbert space. Thus $X$ contains no subspace isomorphic to $c_{0}$ or $l_{1}, X$ is somewhat reflexive, and no nonreflexive subspace has an unconditional basis.
\end{abstract}

It has been conjectured that every infinite-dimensional Banach space has an infinite-dimensional subspace that is either reflexive or isomorphic to $c_{0}$ or to $l_{1}[9, \mathrm{p} .165]$. A counterexample would also be an example of a space that has no infinite-dimensional subspace with an unconditional basis [6, Theorem 2, p. 521]. It is known that there is a nonreflexive Banach space $J$ with no subspace isomorphic to $c_{0}$ or to $l_{1}[6, \mathrm{pp} .523-527]$, but $J^{* *}$ is separable. Each of the following is a necessary and sufficient condition for a separable Banach space $X$ to contain a subspace isomorphic to $l_{1}$; separability is not needed for conditions (i) and (ii) (see [5, Theorem 2.1, p. 13] and $[10$, p. 475]).

(i) $L_{1}[0,1]$ is isomorphic to a subspace of $X^{*}$.

(ii) $C[0,1]^{*}$ is isomorphic to a subspace of $X^{*}$.

(iii) $X^{*}$ has a subspace isomorphic to $l_{1}(\Gamma)$ for some uncountable $\Gamma$.

A natural and well-known conjecture in view of the preceding is that a Banach space has a subspace isomorphic to $l_{1}$ if the space is separable and its dual is not separable (e.g., see [1, $\S 9$, p. 243], [2, $§ 5.4$, p. 174], and the last paragraph of [11]). It will be shown that this conjecture is false. The counterexample $\tilde{X}$ has the property that each infinite-dimensional subspace has an infinite-dimensional subspace isomorphic to Hilbert space. Thus $\tilde{X}$ is also a counterexample to the conjecture that each separable somewhat-reflexive space has a separable dual (see [3, Problem 3, p. 191] and [8, Remark IV.2, p. 86]). Also, neither $c_{0}$ nor $l_{1}$ has an infinitedimensional subspace isomorphic to Hilbert space, so no nonreflexive subspace has an unconditional basis [6, Theorem 2, p. 521]. It has been

AMS (MOS) subject classifications (1970). Primary 46B10.

${ }^{1}$ Supported in part by NSF Grant GP-28578.

Copyright @ American Mathematical Society 1974 
shown by J. Lindenstrauss and C. Stegall that $X$ is a counterexample for several other conjectures. They will present these results in a later paper, as well as giving another example of a separable space with nonseparable dual that has no subspace isomorphic to $l_{1}$ (this space has a subspace isomorphic to $c_{0}$ ).

The counterexample is intimately related to the space $J$ mentioned above, e.g., many complemented subpsaces are isometric to $J$. This follows from the fact that, if $x=\left\{x_{i}\right\} \in J$, then

$$
\|x\|=\sup \left\{\left[\sum_{i=1}^{n-1}\left(x_{p(i+1)}-x_{p(i)}\right)^{2}\right]^{1 / 2}: 1 \leqq p(1)<\cdots<p(n), n \geqq 1\right\},
$$

but if $x$ is written as $\left\{\xi_{i}\right\}$, where $\xi_{i}=x_{i}-x_{i+1}$ and $x=\sum_{1}^{\infty} \xi_{i} e_{i}$ with $e_{n}$ the sequence for which $x_{i}=1$ if $i \leqq n$ and $x_{i}=0$ if $i>n$, then

$$
\|x\|=\sup \left\{\left[\sum_{j=1}^{n-1}\left(\sum_{i=p(j)}^{p(j+1)-1} \xi_{i}\right)^{2}\right]^{1 / 2}: 1 \leqq p(1)<\cdots<p(n), n \geqq 1\right\} .
$$

To describe the counterexample, first choose a set $\Omega$ of cardinality $c$, each of whose members can be thought of as an infinite subset of the positive integers or as a nested sequence of intervals obtained as follows. Associate $1=b(1,1)$ with the interval $[0,1]$, then $2=b(2,1)$ with $\left[0, \frac{1}{3}\right]$ and $3=b(2,2)$ with $\left[\frac{2}{3}, 1\right]$, and in general for each positive integer $n$ use the integers from $2^{n-1}$ to $2^{n}-1$, or $\left\{b(n, i): 1 \leqq i \leqq 2^{n-1}\right\}$, to label the $2^{n-1}$ intervals remaining at the $n$th stage of the process customarily used to describe the Cantor set. Now let each number $t$ in the Cantor set determine a member of $\Omega$, namely, the set of all integers associated with intervals containing $t$. By a segment we shall mean a finite increasing sequence (possibly empty) of consecutive members of some set $\mathscr{A} \in \Omega$. If $\mathscr{A} \neq \mathscr{B}$ and $\mathscr{A}$ and $\mathscr{B}$ are in $\Omega$, then $\mathscr{A} \cap \mathscr{B}$ is a nonempty initial segment of both $\mathscr{A}$ and $\mathscr{B}$. A branch point of order $k$ for $\Omega$ is one of the integers $\{b(k, i)$ : $\left.1 \leqq i \leqq 2^{k-1}\right\}$ that is the $k$ th term of some member of $\Omega$. A branch of order $k$ (or a $k$-branch) is an infinite increasing sequence of consecutive members of some set $\mathscr{A} \in \Omega$ whose first member is a branch point of order $k$. For each sequence $x=\left\{x_{i}\right\}$ of real numbers with finite support, let

$$
\|x\|=\sup \left\{\left[\sum_{n}\left(\sum_{i \in A(n)} x\right)^{2}\right]^{1 / 2}\right\}
$$

where the sup is over all finite sets $\{A(n): 1 \leqq n \leqq p\}$ of pairwise disjoint segments. Let $\tilde{X}$ be the completion with respect to this norm of the normed linear space $X$ of all such sequences. Then $\tilde{X}$ is a separable Banach space.

For each $\mathscr{A} \in \Omega$, define a linear functional $f_{\mathscr{A}}$ on $X$ by letting $f_{\mathscr{A}}(x)=$ $\sum_{i \in \mathscr{A}} x_{i}$ if $x \in X$, and extending to $\tilde{X}$ by use of continuity. Then $\left\|f_{\mathscr{A}}\right\|=1$. 
Also, if $m \in \mathscr{A}-\mathscr{B}, n \in \mathscr{B}-\mathscr{A}$, and $x$ is the sequence $\left\{x_{i}\right\}$ with $x_{m}=1$, $x_{n}=-1$ and $x_{i}=0$ otherwise, then $\left(f_{\mathscr{A}}-f_{\mathscr{O}}\right)(x)=2$ and $\|x\|=2^{1 / 2}$. Thus $\left\|f_{\mathscr{A}}-f_{\mathscr{B}}\right\| \geqq 2^{1 / 2}$ and $\tilde{X}^{*}$ is not separable.

THEOREM. If $\theta>\sqrt{ } 2$, then each infinite-dimensional subspace of $\tilde{X}$ contains an infinite-dimensional subspace $H$ for which there is an inner-product norm ||| || such that

$$
\|x\| \leqq\|x\| \leqq \theta\|x\| \| \text { if } x \in H .
$$

Proof. It is sufficient to prove the theorem for $X$. Let $Y$ be an infinitedimensional subspace of $X$ and let $Y^{k}$ be the subspace of $Y$ whose members are zero at each of the finite set of branch points with order less than $k$. Then $Y^{k}$ has finite codimension as a subspace of $Y$. For each $x$ in $X$, let

$$
[x]_{k}=\sup \left\{\left[\sum_{n}\left(\sum_{i \in B(n)} x_{i}\right)^{2}\right]^{1 / 2}\right\},
$$

where the sup is over all sets $\{B(n)\}$ of pairwise disjoint $k$-branches. Let

$$
\omega=\liminf _{k \rightarrow \infty}\left\{[x]_{k}: x \in Y^{k} \text { and }\|x\|=1\right\} .
$$

It will be shown that $\omega=0$. Suppose $\omega>0$. For $\varepsilon>0$, choose $K$ so that

$$
\inf \left\{[x]_{k}^{2}: x \in Y^{k} \text { and }\|x\|=1\right\}>\omega^{2}-\varepsilon \text { if } k \geqq K .
$$

Choose an increasing sequence of integers $\{m(k)\}$ with $m(1)=K$, and then a sequence $\left\{y^{k}\right\}$ in $X$ such that, for each $k,\left\|y^{k}\right\|=1, y^{k}$ has nonzero terms only at branch points with orders in the interval $[m(k), m(k+1))$, and

$$
\left[y^{k}\right]_{m(k)}^{2}<\omega^{2}+\varepsilon .
$$

It will be shown that a contradiction is obtained if $\varepsilon$ is sufficiently small. Let $y^{k}=\left\{y_{i}^{k}\right\}$. Since $\left[y^{k}\right]_{K}^{2}>\omega^{2}-\varepsilon$ and $y^{k} \in Y^{m(k)}$, there are $2^{K-1}$ branch points of order $m(k)$, which will be denoted by $\left\{b\left(k, p_{i}^{k}\right): 1 \leqq i \leqq 2^{K-1}\right\}$ rather than using $b\left(m(k), p_{i}^{k}\right)$, and $2^{K-1}$ branches $\left\{B\left(k, p_{i}^{k}\right): 1 \leqq i \leqq 2^{K-1}\right\}$ of order $m(k)$ starting at these branch points, such that

$$
\sum_{i}\left(\sum_{j \in B\left(k, p_{i}{ }^{k}\right)} y_{j}^{k}\right)^{2}>\omega^{2}-\varepsilon
$$

Now for each $i, k$ and $\kappa$ with $i \leqq 2^{K-1}$ and $k<\kappa$, let $\sigma(k, \kappa ; i)$ be $j$ if there exists $j \leqq 2^{K-1}$ such that $b\left(k, p_{i}^{k}\right)$ and $b\left(\kappa, p_{j}^{\kappa}\right)$ are on the same $K$ branch. Then $\sigma(k, \kappa ; i)$ is strictly increasing as a function of $i$ and determines a one-to-one mapping of a subset of $\left\{p_{i}^{k}: i \leqq 2^{K-1}\right\}$ onto a subset of $\left\{p_{i}^{\kappa}: i \leqq 2^{K-1}\right\}$. Choose a sequence of positive integers $I_{1}$ so that if $k$ and $\kappa$ are in $I_{1}$ and $k<\kappa$, then $\sigma(k, \kappa ; i)=\sigma(k ; i)$ is independent of $\kappa$ for each $i$. 
Then choose a subsequence $I_{2}$ of $I_{1}$ so that if $k \in I_{2}$ then $\sigma(k ; i)=\sigma(i)$ is independent of $k$ for each $i$. Now, $\sigma[\sigma(i)]=\sigma(i)=i$ and, for each $i \leqq 2^{K-1}$, either $i$ is in the domain of $\sigma$ and there is a $K$-branch that contains all $b\left(k, p_{i}^{k}\right)$ for $k \in I_{2}$, or $i$ is not in the domain of $\sigma$ and no $K$-branch that contains $b\left(k, p_{i}^{k}\right)$ for some $k \in I_{2}$ can contain any $b\left(\kappa, p_{j}^{\kappa}\right)$ for $\kappa \neq k$ and $\kappa \in I_{2}$.

Now choose a subsequence $I_{3}$ of $I_{2}$ such that, for each $i$ in the domain of $\sigma$ and any two members $k$ and $\kappa$ of $I_{3}$,

$$
\left|\sum_{i \in B} y_{i}^{k}-\sum_{i \in B} y_{i}^{\kappa}\right|<2^{-K / 2} \varepsilon
$$

where $B$ is the $K$-branch containing all $b\left(k, p_{i}^{k}\right)$ for $k \in I_{3}$. For a $\lambda$ to be chosen later, let $\{\mu(j): 1 \leqq j \leqq \lambda\}$ be any $\lambda$ consecutive members of $I_{3}$ and, for any $K$-branch $B$, consider

$$
\left[\sum_{i \in B}\left(\sum_{j=1}^{\lambda}(-1)^{j} y_{i}^{\mu(j)}\right)\right]^{2}=\left[\sum_{j=1}^{\lambda}(-1)^{j}\left(\sum_{i \in B} y_{i}^{\mu(j)}\right)\right]^{2} .
$$

For each $\mu(j)$, let $\sum_{i \in B} y_{i}^{\mu(j)}$ be denoted by $\rho_{B}^{\mu(j)}$ or $\Delta_{B}^{\mu(j)}$ accordingly as $B$ contains one of the branch points $\left\{b\left[\mu(j), p_{i}^{\mu(j)}\right]\right\}$ or $B$ does not contain any such branch point. Then either there exists $\iota \leqq 2^{K-1}$ and $\kappa>0$ such that $B$ contains $\left\{b\left(\mu(j), p_{t}^{\mu(j)}\right): j \leqq \kappa\right\}$ and $B$ contains no other $b\left(\mu(j), p_{i}^{\mu(j)}\right)$ for $\kappa<j \leqq \lambda$ and $i \leqq 2^{K-1}$, or else $B$ contains at most one of $\left\{b\left[\mu(j), p_{i}^{\mu(j)}\right]\right.$ : $\left.1 \leqq j \leqq \lambda, i \leqq 2^{K-1}\right\}$. For any real numbers $\left\{a_{i}\right\}$,

$$
\left(\sum_{1}^{n} a_{i}\right)^{2} \leqq \sum_{1}^{n} 2^{i} a_{i}^{2}
$$

Therefore it follows from (4) that the expression (5) is not greater than

$$
2\left(\rho_{B}^{\mu}\right)^{2}+4\left(\left[\frac{\kappa}{2}\right] 2^{-K / 2} \varepsilon\right)^{2}+\sum_{j=1}^{\lambda} \varepsilon_{j} 2^{\lambda}\left(\Delta_{B}^{\mu(j)}\right)^{2},
$$

where $\mu \in\{\mu(j): j \leqq \lambda\}$ (except that the first term in (7) may be missing), $\kappa$ is the largest integer such that $\kappa \leqq \lambda$ and $B$ contains $b\left(\mu(j), p_{t}^{\mu(j)}\right)$ for some $\iota$ and for all $j \leqq \kappa$, and each $\varepsilon_{j}$ is 0 or 1 . Note that if we sum terms of type $\left(\Delta_{B(n)}^{\mu(j)}\right)^{2}$ over any $2^{K-1}$ pairwise disjoint $K$-branches $B(n)$, then it follows from (2) and (3) that this sum is not greater than $2 \varepsilon$; also, there are then at most $2^{K-1}$ terms of the type of the first term in (7), so these can contribute to $\left[y^{\mu(j)}\right]_{m[\mu(j)]}^{2}$ for at most $2^{K-1}$ values of $j$. Therefore the sum of (5) or (7) over any $2^{K-1}$ pairwise disjoint $K$-branches is not greater than

$2 \cdot 2^{K-1}\left(\omega^{2}+\varepsilon\right)+4[\lambda / 2] \varepsilon^{2}+\lambda \cdot 2^{\lambda}(2 \varepsilon) \leqq 2^{K} \omega^{2}+\varepsilon\left(2^{K}+\lambda \cdot 2^{\lambda+1}\right)+2 \lambda \varepsilon^{2}$. 
Since $\left\|\sum_{j=1}^{\lambda}(-1)^{j} y^{\mu(j)}\right\|^{2} \geqq \lambda$, this contradicts (1) if

$$
2^{K} \omega^{2}+\varepsilon\left(2^{K}+\lambda \cdot 2^{\lambda+1}\right)+2 \lambda \varepsilon^{2}<\lambda\left(\omega^{2}-\varepsilon\right) .
$$

This inequality can be satisfied by choosing $\lambda>2^{K}$ and then choosing $\varepsilon$ small enough.

This concludes the proof that $\omega=0$. Since $\omega=0$, we can let $\varepsilon$ be a positive number and choose an increasing sequence of integers $\{n(k)\}$ and a sequence $\left\{y^{k}\right\}$ in $X$, such that, for each $k,\left\|y^{k}\right\|=1, y^{k}$ has nonzero terms only at branch points with orders in the interval $(n(k), n(k+1))$, and

$$
\left[y^{k}\right]_{n(k)}^{2}<2^{-k} \varepsilon^{2} .
$$

Let $\left\{a_{i}\right\}$ be a finite sequence of real numbers with $\sum a_{i}^{2}>0$. Then $\left\|\sum a_{j} y^{j}\right\|^{2} \geqq$ $\sum a_{i}^{2}$. Choose a finite set $\{A(n): 1 \leqq n \leqq p\}$ of pairwise disjoint segments such that

$$
\left\|\sum a_{j} y^{j}\right\|^{2}=\sum_{n}\left(\sum_{i \in A(n)} \sum_{j} a_{j} y_{i}^{j}\right)^{2} .
$$

If $A$ is any of these segments, then $A$ is the union of an initial and a terminal segment, each of which contains a part of the piece of a branch between branch points of order $n(j)$ and branch points of order $n(j+1)$ for some $j$, and several interior segments, each of which has the property that there is a $j$ such that the segment contains all of the piece of a branch between branch points of order $n(j)$ and branch points of order $n(j+1)$. A sum $\sum_{j} a_{j} y_{i}^{j}$ over those $i$ in an initial segment or a sum over those $i$ in a terminal segment contributes only to the norm of the corresponding $a_{j} y^{j}$, while a sum over an interior segment contributes to $\left[a_{j} y^{j}\right]_{n(j)}$ only. Now we can use the fact that

$$
(a+b+c)^{2} \leqq(2+\varepsilon)\left(a^{2}+b^{2}\right)+(1+2 / \varepsilon) c^{2}
$$

for any real numbers $a, b$ and $c$, and then (6) and (8), to obtain

$$
\begin{aligned}
\left\|\sum a_{j} y^{j}\right\|^{2} & \leqq(2+\varepsilon) \sum a_{j}^{2}+\left(1+\frac{2}{\varepsilon}\right) \sum_{j} 2^{j} a_{j}^{2}\left[y^{j}\right]_{n(j)}^{2} \\
& <(2+\varepsilon) \sum a_{j}^{2}+\left(2 \varepsilon+\varepsilon^{2}\right) \sum a_{j}^{2}=\left(2+3 \varepsilon+\varepsilon^{2}\right) \sum a_{j}^{2} .
\end{aligned}
$$

Since $\varepsilon$ was arbitrary, for any $\theta>\sqrt{ } 2$ there is an infinite sequence $\left\{y^{k}\right\}$ of members of $X$ such that, for all sequences $\left\{a_{i}\right\}$ of real numbers,

$$
\left(\sum_{1}^{\infty} a_{i}^{2}\right)^{1 / 2} \leqq\left\|\sum_{1}^{\infty} a_{i} y^{i}\right\| \leqq \theta\left(\sum_{1}^{\infty} a_{i}^{2}\right)^{1 / 2}
$$


ERRATUM ADDED IN PROOF. The sequences $\{m(k)\}$ and $\left\{y^{k}\right\}$ should be chosen simultaneously so that each $y^{k}$ is in $Y$ and the branches $\left\{B\left(k, p_{i}^{k}\right)\right.$ : $\left.1 \leqslant i \leqslant 2^{K-1}\right\}$ are pieces of pairwise disjoint $K$-branches; $\{\mu(j): 1 \leqslant j \leqslant \lambda\}$ should not be consecutive members of $I_{3}$, but chosen so that, for each branch point $b$ of order $K$,

$$
\sup \left\{\left[\sum_{i \in B} y_{i}^{\mu(j)}\right]^{2}\right\}<\sup \left\{\left[\sum_{i \in B} y_{i}^{\mu(1)}\right]^{2}\right\}+2^{1-K} \epsilon,
$$

where $1 \leqslant j \leqslant \lambda$ and $B$ is any $K$-branch containing $b$. In the first term of (7), $\rho_{B}^{\mu}$ should be replaced by the sum of the absolute values of two such terms; in the next two inequalities, $2^{K}\left(\omega^{2}+\epsilon\right)$ can now be replaced by $8 \omega^{2}+16 \epsilon$ and $\lambda$ need not depend on $K$.

\section{REFERENCES}

1. S. Banach, Théorie des opérations linéaires, Monografie Mat., PWN, Warsaw, 1932.

2. C. Bessaga and A. Pelczyński, A generalization of results of $R$. C. James concerning absolute bases in Banach spaces, Studia Math. 17 (1958), 165-174. MR 22 \#5874.

3. W. J. Davis and I. Singer, Boundedly complete M-bases and complemented subspaces in Banach spaces, Trans. Amer. Math. Soc. 175 (1973), 187-194. 42.

4. J. Hagler, Some more Banach spaces which contain $l^{1}$, Studia Math. 46 (1973), 35-

5. —, Embeddings of $L^{1}$ spaces into conjugate Banach spaces, Ph.D. thesis (U.C.B.), 1972.

6. R. C. James, Bases and reflexivity of Banach spaces, Ann. of Math. (2) 52 (1950), 518-527. MR 12, 616.

7. —, Uniformly non-square Banach spaces, Ann. of Math. (2) 80 (1964), 542550. MR 30 \#4139.

8. W. B. Johnson and H. P. Rosenthal, On $w^{*}$-basic sequences and their applications to the study of Banach spaces, Studia Math. 43 (1972), 77-92.

9. J. Lindenstrauss, Some aspects of the theory of Banach spaces, Advances in Math. 5 (1970), 159-180. MR 43 \#5288.

10. C. Stegall, Banach spaces whose duals contain $l_{1}(\Gamma)$ with applications to the study of dual $L_{1}(\mu)$ spaces, Trans. Amer. Math. Soc. 176 (1973), 463-477.

11. - The Radon-Nikodym property in conjugate Banach spaces, Trans. Amer. Math. Soc. (to appear).

Department of Mathematics, Claremont Graduate School, Claremont, California 91711 